\title{
A Retrospective Study of the Effects of Psychotropic Drugs on Neuroendocrine Hormones in Patients with Bipolar Disorder
}

\author{
Shengnan Zhao \\ Bo Zhang \\ Xueli Sun
}

Mental Health Center, West China Hospital of Sichuan University, Chengdu, Sichuan, People's Republic of China
Correspondence: Xueli Sun Mental Health Center, West China Hospital of Sichuan University, Chengdu, Sichuan, People's Republic of China Tel +86-17323085880

Email sunxuelihx@163.com
Background: Bipolar disorder (BD) is predominantly treated with medication, and previous studies have shown that psychotropic drugs have an effect on the neuroendocrine system. And the objectives of this study were: 1) to assess changes in neuroendocrine hormones of HPA axis and HPT axis in patients with BD after psychotropic drug treatment; 2) to determine differences in the above neuroendocrine hormones between psychotropic drug monotherapy and polytherapy treated patients.

Methods: This study was a retrospective study that included 188 subjects.

Results: After psychotropic drugs treatment, TSH levels increased significantly from baseline $(\mathrm{p}<0.001)$ and FT3, TT3, FT4, TT4, ACTH and CORT levels decreased significantly from baseline $(\mathrm{p}<0.001, \mathrm{p}<0.001, \mathrm{p}<0.001, \mathrm{p}=0.007, \mathrm{p}=0.039, \mathrm{p}=0.031)$. There was no significant difference in hormonal changes between the monotherapy group and the polytherapy group, even though the polytherapy group was further divided into 4 subgroups. And there was no correlation between changes in hormones and treatment duration.

Conclusion: Although the incidence of significant HPT axis and HPA axis dysfunction after psychotropic drug treatment is low in patients with BD who have normal baseline neuroendocrine hormone levels, psychotropic drug treatment does suppress HPT axis and HPA axis function irrespective of the use of psychotropic drugs alone or in combination, and duration of psychotropic drug treatment. Therefore, even patients with BD who have normal baseline levels of neuroendocrine hormones should have their neuroendocrine hormone levels regularly monitored after psychotropic drug medication. But, the interval of monitoring can be slightly longer, which can be once every six months after taking psychotropic drugs.

Keywords: bipolar disorder, psychotropic drugs, neuroendocrine hormones

\section{Introduction}

Bipolar disorder (BD) is a chronic, relapsing disorder that affects more than $1 \%$ of the world's population, ${ }^{1}$ and often results in cognitive impairment and reduced quality of life. ${ }^{2,3} \mathrm{BD}$ is a complex medical condition and the etiology is unknown. Previous reports suggest that genetic and epigenetic factors, and environmental stressors combine to contribute to the expression of the disease. ${ }^{4}$ For example, a systematic review shows that experiences of childhood traumatic experiences contribute to non-suicidal self-injury and suicide. ${ }^{5}$ Meanwhile, extreme sensory processing patterns, impulsivity, alexithymia, depression, and hopelessness may show a characteristic pattern in patients with major affective disorders. ${ }^{6}$ 
Studies have shown that BD patients are more likely to develop abnormal thyroid function than healthy population, ${ }^{7-9}$ and many patients with $\mathrm{BD}$ have manifestations of HPA axis hyperactivity, such as hypercortisolism, with an increased non-suppression rate in the Dexamethasone Suppression Test (DST) or the more sensitive Dexamethasone Suppression/Corticotropin-releasing Hormone Stimulation test (DEX/CRH test). ${ }^{10,11}$ In addition, psychotropic drugs have been shown to cause some effects on the neuroendocrine system. As we all know, lithium can inhibit thyroid hormone secretion, leading to an increase in thyroid stimulating hormone (TSH) which can lead to goiter. Previous studies have shown that clinical and subclinical hypothyroidism may appear within a few weeks of starting lithium therapy. ${ }^{12}$ Atypical antipsychotics (AAPs) can also cause some abnormalities in thyroid function based on dopamine antagonism. After atypical antipsychotics treatment, psychiatric patients seemed to have a trend of hypothyroidism. ${ }^{13}$ A prospective, double-blind study that enrolled patients with schizophrenia indicated that after 6 weeks of medication, thyroxine (T4) levels decreased significantly in patients treated with quetiapine. ${ }^{14}$ Besides, it has been reported that the main effect of selective serotonin reuptake inhibitors (SSRIs) on thyroid function is to reduce triiodothyronine (T3) and T4 levels, without affecting TSH levels. ${ }^{15,16}$ And several studies indicated that antipsychotics and antidepressants tend to suppress the secretion of cortisol. ${ }^{17-20}$ In turn, the neuroendocrine hormone levels can affect the patient's response to drug therapy. Thyroid dysfunction is associated with poor treatment response in bipolar depression and mixed states and higher cortisol was associated with SSRI nonresponse. ${ }^{21-23}$

At present, BD is still predominantly treated with medication in clinical practice, and to achieve rapid and effective control of mood symptoms and to reduce relapse, clinicians often use polypharmacy for BD patients. ${ }^{24,25}$ Therefore, a comprehensive understanding of the neuroendocrine effects of psychotropic drugs on patients with bipolar disorder is essential. And, we hypothesized that psychotropic medications decrease neuroendocrine hormone secretion in patients with $\mathrm{BD}$, and this may not be related to psychotropic drug monotherapy and polytherapy. Accordingly, the objectives of this study were: (1) to assess changes in neuroendocrine hormones of HPA axis and HPT axis in patients with BD after psychotropic drug treatment; (2) to determine differences in the above neuroendocrine hormones between psychotropic drug monotherapy and polytherapy treated patients.

\section{Materials and Methods}

The study was approved by the China Ethics Committee of Registering Clinical Trials (ChiECRCT-20180187). Because this was a retrospective, non-interventional study and all data were collected anonymously, informed consent was not required. The study retrospectively evaluated the medical records of inpatients in the Mental Health Center of West China Hospital of Sichuan University from November 2016 to December 2019.

Inclusion criteria: (1) aged 18 to 65 years; (2) had not received psychotropic medication or treatment that may affect the function of the HPT axis or HPA axis for at least 6 months prior to admission; (3) started psychotropic medication within 24 hours of admission and continuing to receive psychotropic medication; (4) had received psychotropic medication for at least 14 days, and did not change the medication regimens within one year. Here were the exclusion criteria: (1) abnormal level of any the seven neuroendocrine hormone indicators at baseline, including 5 indicators of HPT axis, TSH, free triiodothyronine (FT3), total triiodothyronine (TT3), free thyroxine (FT4), and total thyroxine (TT4), and 2 indicators of HPA axis, adrenocorticotrophic hormone (ACTH) and cortical hormone (CORT); (2) received medications known to affect HPA axis and HPT axis function in the study course, for instance, antithyroid medications (prothiouracil, methimazole/thimazole, and carbimazole), levothyroxine, iodine-containing medications, or glucocorticoid medications (cortisone, methylprednisolone, dexamethasone); (3) pregnant or lactating women.

The medical history of each subject was collected completely, including demographic data (gender, age, and so on) and clinical features, such as psychotropic medication (specific medication regimen and duration of medication), and serum neuroendocrine hormone levels (including TSH, FT3, TT3, FT4, TT4, ACTH, and CORT) at baseline and within one year of admission. The last test result of serum TSH, FT3, TT3, FT4, TT4, ACTH, and CORT level was used as serum neuroendocrine hormone level at follow-up.

FT3, TT3, FT4, TT3, TT4 and CORT were measured using quantitative electrochemiluminescence analysis, TSH was measured using electrochemiluminescence double antibody sandwich assay, and ACTH was measured using radioimmunoassay. The normal ranges of serum 
neuroendocrine hormone levels were as follows, TSH: 0.27-4.2mU/L, FT3: 3.60-7.50 pmol/L, TT3: 1.3-3.1 $\mathrm{nmol} / \mathrm{L}$, FT4: 12-22 pmol/L, TT4: 62-164 nmol/L, ACTH: 5-78 ng/L, and CORT: $133-537 \mathrm{nmol} / \mathrm{L}$. The diagnosis was made by psychiatrists in accordance with the International Classification of Diseases, 10th Revision (ICD-10). In the course of hospitalization, blood samples were collected between 7:00 and 8:00 a.m. before breakfast to evaluate serum endocrine hormone levels.

The psychotropic drugs referred to in this study were: mood stabilizers (MSs) (including antiepileptic drugs such as lamotrigine), AAPs, and antidepressants (ADs). When comparing the effects of different treatment regimens on neuroendocrine hormones in patients with bipolar disorder, participants were divided into the monotherapy group (one of the three classes of drugs mentioned above alone) and the polytherapy group (a combination of two or three of the three classes of drugs mentioned above). For further comparison, the polytherapy group was subdivided into four subgroups as follows: $\mathrm{AD}+\mathrm{AAP}, \mathrm{AD}+\mathrm{MS}, \mathrm{AAP}$ $+\mathrm{MS}, \mathrm{AD}+\mathrm{AAP}+\mathrm{MS}$.

Categorical variables are reported as numbers (percentages) and continuous variables are reported as medians (first quartile $=\mathrm{Q} 1$, third quartile $=\mathrm{Q} 3$ ). The Mann-Whitney $U$-test or Kruskal-Wallis test was used to compare demographic and clinical data between subgroups for continuous variables and the chi-squared test for categorical variables. The Wilcoxon matched-pairs rank test was used to compare serum endocrine hormone levels at baseline and after psychotropic medication. The Spearman rank correlation test was used to evaluate the correlation between the changes in HPA axis hormones and HPT axis hormones, as well as the correlation between the changes in the seven kinds of serum neuroendocrine hormones and the treatment time of psychotropic drug. Data were analyzed using two-tailed significance estimates. Statistically significant $\mathrm{p}$-values were set at $<0.05$. All statistical analyses were conducted using the SPSS 24.0 software (Statistical Package for Social Sciences, SPSS Inc).

\section{Results}

Demographic and clinical data of the participants and the differences between groups are shown in Table 1. The specific medication regimen is shown in Appendix 1. The study ultimately included 188 patients with BD, of whom, 119 bipolar depression, 48 bipolar mania, and 21 not classified. In general, 98 (53.13\%) were male and 90 $(47.87 \%)$ were female, age at enrollment was 36.50
$(27.00,44.00)$ years, age at first presentation was 28.00 $(21.00,36.00)$ years, and the duration of this treatment was $157.50(70.00,257.00)$ days. There were $26(13.83 \%)$ in the monotherapy group (MS:14; AAP:12) and 162 $(86.17 \%)$ in the polytherapy group (AD+AAP: 30, AD +MS: 32, AAP+MS: 34, AD+AAP+MS: 66). Antidepressants were used by 128 (68.09\%) people, most often paroxetine (28.13\%); $146(77.66 \%)$ people used mood stabilizers, most often valproate (34.25\%); 140 (74.47\%) people used atypical antipsychotics, most often olanzapine (37.14\%) and quetiapine (37.14\%). There were no significant differences between the monotherapy group and the polytherapy group in terms of gender, age at entry, and duration of current treatment, even though the polytherapy group was further divided into 4 subgroups (AD $+\mathrm{AAP}, \mathrm{AD}+\mathrm{MS}, \mathrm{AAP}+\mathrm{MS}$, and $\mathrm{AD}+\mathrm{AAP}+\mathrm{MS})$.

The changes in serum TSH, FT3, TT3, FT4, TT4, $\mathrm{ACTH}$, and CORT levels before and after psychotropic drug medication are presented in Table 2, as well as the comparisons between the monotherapy group and the polytherapy group. It can be found that, after psychotropic drug treatment, TSH levels increased significantly from baseline $(\mathrm{p}<0.001)$ and FT3, TT3, FT4, TT4, ACTH and CORT levels decreased significantly from baseline $(\mathrm{p}<0.001, \quad \mathrm{p}<0.001, \quad \mathrm{p}<0.001, \quad \mathrm{p}=0.007, \quad \mathrm{p}=0.039$, $\mathrm{p}=0.031$ ). However, there was no significant difference in hormonal changes between the monotherapy group and the polytherapy group, even though the polytherapy group was further divided into 4 subgroups. Besides, TSH levels in 9 patients and TT3 levels in 2 patients were all higher than the normal range. The TT3 levels in 25 patients, FT3 levels in 18 patients, TT4 levels in 12 patients, FT4 levels in 19 patients, ACTH levels in 1 patient, and CORT levels in 4 patients were all below the normal ranges. And only 4 patients developed overt hypothyroidism.

Last, the Spearman rank correlation test found no correlation between changes in hormones and psychotropic drug medication time. And there was no correlation between changes in neuroendocrine hormones of the HPA axis and the HPT axis.

\section{Discussion}

The study found that TSH levels were significantly increased and FT3, TT3, FT4, TT4, ACTH and CORT levels were significantly decreased after psychotropic drug treatment. But, only four individuals eventually developed hypothyroidism. Those results indicated that HPT function and HPA axis function were suppressed 
Table I Demographic and Clinical Data

\begin{tabular}{|c|c|c|c|c|c|c|c|c|c|}
\hline & \multirow[t]{2}{*}{ Total } & \multirow[t]{2}{*}{ Monotherapy } & \multicolumn{5}{|l|}{ Polytherapy } & \multirow[t]{2}{*}{$\mathbf{p}^{\mathbf{a}}$} & \multirow[t]{2}{*}{$\mathbf{p}^{\mathbf{b}}$} \\
\hline & & & Total & AD+AAP & $A D+M S$ & AAP+MS & $\begin{array}{l}\text { AD+AAP } \\
+M S\end{array}$ & & \\
\hline \multicolumn{10}{|l|}{ Gender } \\
\hline Male & $98(52.13)$ & $10(38.46)$ & $88(54.32)$ & $17(56.67)$ & $13(40.63)$ & $16(47.06)$ & $32(48.48)$ & 0.358 & 0.651 \\
\hline Female & $90(47.87)$ & $16(61.54)$ & $74(45.68)$ & $13(43.33)$ & $19(59.37)$ & $18(52.94)$ & $34(51.52)$ & & \\
\hline \multicolumn{10}{|l|}{ Educational background } \\
\hline Primary school and below & $62(32.98)$ & $9(34.62)$ & $53(32.72)$ & $9(30.00)$ & $12(37.50)$ & $12(35.29)$ & $20(30.30)$ & 0.663 & 0.764 \\
\hline Junior high school & $35(18.62)$ & $3(11.54)$ & $32(19.75)$ & $4(13.33)$ & $6(18.75)$ & $4(11.76)$ & $18(27.27)$ & & \\
\hline Senior high school & $39(20.74)$ & $5(19.23)$ & $34(20.99)$ & $6(20.00)$ & $6(18.75)$ & $8(23.53)$ & $14(21.21)$ & & \\
\hline Junior college or bachelor & $36(19.15)$ & $7(26.92)$ & $29(17.90)$ & $8(26.67)$ & $6(18.75)$ & $4(11.76)$ & II (16.67) & & \\
\hline Postgraduate or above & $17(9.04)$ & $2(7.69)$ & $14(8.64)$ & $3(10.00)$ & $2(6.25)$ & $6(17.65)$ & $3(4.55)$ & & \\
\hline \multicolumn{10}{|l|}{ Marital status } \\
\hline Unmarried & 66 (35.11) & $8(30.77)$ & $58(35.80)$ & $8(26.67)$ & $12(37.50)$ & $10(29.4 I)$ & $28(42.42)$ & 0.288 & 0.356 \\
\hline Married & $98(52.13)$ & $12(46.15)$ & $86(53.09)$ & $20(66.67)$ & $18(56.25)$ & $18(52.94)$ & $30(45.45)$ & & \\
\hline Divorced & $24(12.76)$ & $6(23.07)$ & $18(11.11)$ & $2(6.66)$ & $2(6.25)$ & $6(17.65)$ & $8(12.12)$ & & \\
\hline \multicolumn{10}{|l|}{ Occupation } \\
\hline $\begin{array}{l}\text { Unemployed (including } \\
\text { retirement) }\end{array}$ & & $8(30.77)$ & $74(45.68)$ & $10(33.33)$ & $14(43.75)$ & $20(58.82)$ & & 0.155 & 0.176 \\
\hline Student or on job & $106(56.38)$ & $18(69.23)$ & $88(54.32)$ & $20(66.67)$ & $18(56.25)$ & $14(41.18)$ & $36(54.55)$ & & \\
\hline \multicolumn{10}{|l|}{ Family history } \\
\hline No & $158(84.04)$ & $24(92.31)$ & $134(7 \mid .28)$ & $25(83.33)$ & $27(84.38)$ & $28(82.35)$ & $54(81.82)$ & 0.181 & 0.754 \\
\hline Yes & $30(15.96)$ & $2(7.69)$ & $28(18.72)$ & $5(16.67)$ & $5(15.62)$ & $6(17.65)$ & $12(18.18)$ & & \\
\hline Age at enrolment (years) & $\begin{array}{l}36.50 \\
(27.00,44.00)\end{array}$ & $\begin{array}{l}27.00 \\
(25.75,45.00)\end{array}$ & $\begin{array}{l}36.00 \\
(27.00,44.25)\end{array}$ & $\begin{array}{l}39.00 \\
(27.00,43.00)\end{array}$ & $\begin{array}{l}37.00 \\
(25.75,51.50)\end{array}$ & $\begin{array}{l}38.00 \\
(28.75,47.75)\end{array}$ & $\begin{array}{l}35.00 \\
(24.00,41.25)\end{array}$ & 0.816 & 0.382 \\
\hline Age of first onset (years) & $\begin{array}{l}28.00 \\
(21.00,36.00)\end{array}$ & $\begin{array}{l}32.00 \\
(19.00,41.75)\end{array}$ & $\begin{array}{l}27.00 \\
(21.75,35.25)\end{array}$ & $\begin{array}{l}27.00 \\
(26.00,42.00)\end{array}$ & $\begin{array}{l}27.00 \\
(20.50,42.75)\end{array}$ & $\begin{array}{l}28.00 \\
(25.00,38.25)\end{array}$ & $\begin{array}{l}26.00 \\
(20.00,34.00)\end{array}$ & 0.316 & 0.361 \\
\hline $\begin{array}{l}\text { Duration of this } \\
\text { treatment(ddys) }\end{array}$ & $\begin{array}{l}157.50 \\
(70.00,257.00)\end{array}$ & $\begin{array}{l}202.00 \\
(48.00,288.25)\end{array}$ & $\begin{array}{l}148.00 \\
(73.00,252.75)\end{array}$ & $\begin{array}{l}143.00 \\
(89.00,246.00)\end{array}$ & $\begin{array}{l}183.00 \\
(74,288.75)\end{array}$ & $\begin{array}{l}181.00 \\
(82.50,293.00)\end{array}$ & $\begin{array}{l}116.00 \\
(69.00,233.00)\end{array}$ & 0.504 & 0.545 \\
\hline
\end{tabular}

Notes: ${ }^{a}$ Difference between the monotherapy group and the polytherapy group; ${ }^{b}$ Difference between the monotherapy group, the $A D+A A P$ group, the $A D+M S$ group, the $A A P+M S$ group, and the $A D+A A P+M S$ group.

Abbreviations: $A D$, antidepressant; AAP, atypical antipsychotic; $M S$, mood stabilizer.

after psychotropic drug treatment. In other words, for BD patients with normal baseline neuroendocrine hormone levels, psychotropic drugs may influence thyroid hormones, ACTH, and CORT levels without having major clinical implications on HPT and HPA function. And this is consistent with some previous studies. A nested, matched case-control study indicated that lithium, carbamazepine, and valproate may increase the risk for hypothyroidism, especially when combined. ${ }^{26}$ Another study found that in bipolar depressed patients, one month of treatment with quetiapine alone resulted in a significant decrease in TT4, FT4, and FT3 levels and a significant increase in TSH levels, while TT3 levels did not change significantly. ${ }^{27}$ In contrast, there are relatively few studies on the effects of psychotropic drugs on HPA axis function in patients with BD. A review focused on measuring changes in cortisol before and after drug treatment found that the results of most studies of antipsychotics and antidepressants (except fluoxetine) indicated a reduction of cortisol secretion, ${ }^{28}$ which findings in line with ours.

Stress exposure has been reported to be associated with many mental illnesses, including psychosis, mood disorders, and anxiety disorders. And it is generally known that HPA axis and HPT axis play a role in mediating the relation of stress with psychiatric symptoms. From the results of this study, psychotropic drugs inhibited the activity of the HPA axis and the HPT axis. In other words, psychotropic drugs alleviated the endogenous stress response, thus reducing psychotic symptoms in patients. The indices of HPA axis function are associated 
Table 2 Changes in Serum of TSH, FT3, TT3, FT4, TT4, CORT and ACTH from Baseline After Psychotropic Drugs Medication

\begin{tabular}{|c|c|c|c|c|c|c|}
\hline & $\mathbf{N}$ & Baseline & $\mathbf{p}$ & Changes & $\mathbf{p}^{c}$ & $\mathbf{p}$ \\
\hline \multicolumn{7}{|l|}{ TSH (mU/L) } \\
\hline Total & 188 & $2.26(1.85,3.02)$ & & $0.42(-0.35,0.95)$ & $<0.001$ & \\
\hline Monotherapy & 26 & $2.56(1.88,3.16)$ & $0.369^{\mathrm{a}}$ & $0.08(-0.32,0.54)$ & 0.347 & $0.250^{\mathrm{a}}$ \\
\hline Polytherapy & 162 & $2.22(1.83,2.95)$ & $0.910^{\mathrm{b}}$ & $0.53(-0.35,1.05)$ & $<0.001$ & $0.666^{\mathrm{b}}$ \\
\hline$A D+A A P$ & 30 & $2.44(I .64,3.2 I)$ & & $0.29(-0.63,0.88)$ & 0.379 & \\
\hline$A D+M S$ & 32 & $2.25(1.81,2.76)$ & & $0.39(-0.44,1.26)$ & 0.215 & \\
\hline $\mathrm{AAP}+\mathrm{MS}$ & 34 & $2.37(1.05,3.06)$ & & $0.42(-0.28,1.13)$ & 0.088 & \\
\hline$A D+A A P+M S$ & 66 & $2.13(1.90,2.82)$ & & $0.75(-0.25,1.06)$ & 0.008 & \\
\hline \multicolumn{7}{|l|}{ FT3 (pmol/L) } \\
\hline Total & 188 & $4.87(4.34,5.29)$ & & $-0.40(-1.14,0.15)$ & $<0.001$ & \\
\hline Monotherapy & 26 & $4.76(4.42,5.35)$ & $0.943^{\mathrm{a}}$ & $-0.28(-3.26,0.44)$ & 0.209 & $0.900^{\mathrm{a}}$ \\
\hline Polytherapy & 162 & $4.90(4.33,5.30)$ & $0.573^{\mathrm{b}}$ & $-0.44(-1.09,0.15)$ & $<0.001$ & $0.965^{\mathrm{b}}$ \\
\hline$A D+A A P$ & 30 & $4.95(4.02,5.46)$ & & $-0.55(-1.08,0.14)$ & 0.031 & \\
\hline$A D+M S$ & 32 & $4.44(4.15,5.05)$ & & $-0.39(-0.88,0.22)$ & 0.079 & \\
\hline $\mathrm{AAP}+\mathrm{MS}$ & 34 & $4.90(4.16,5.23)$ & & $-0.82(-1.34,0.16)$ & 0.028 & \\
\hline$A D+A A P+M S$ & 66 & $4.83(4.53,5.4 I)$ & & $-0.41(-1.17,0.13)$ & 0.002 & \\
\hline \multicolumn{7}{|l|}{ TT3 (nmol/L) } \\
\hline Total & 188 & $1.77(1.54,2.14)$ & & $-0.26(-0.75,-0.01)$ & $<0.001$ & \\
\hline Monotherapy & 26 & $1.69(1.52,2.28)$ & $0.776^{a}$ & $-0.15(-1.05,0.35)$ & 0.116 & $0.996^{\mathrm{a}}$ \\
\hline Polytherapy & 162 & $1.79(1.55,2.12)$ & $0.393^{b}$ & $-0.26(-0.69,-0.01)$ & $<0.001$ & $0.962^{b}$ \\
\hline$A D+A A P$ & 30 & $1.76(1.47,2.12)$ & & $-0.17(-0.98,0.16)$ & 0.140 & \\
\hline$A D+M S$ & 32 & $1.94(1.65,2.17)$ & & $-0.29(-0.72,-0.03)$ & 0.021 & \\
\hline$A A P+M S$ & 34 & $2.10(1.68,2.38)$ & & $-0.37(-0.77,0.16)$ & 0.026 & \\
\hline$A D+A A P+M S$ & 66 & $1.72(1.54,2.08)$ & & $-0.26(-0.69,-0.05)$ & 0.001 & \\
\hline \multicolumn{7}{|l|}{ FT4 (pmol/L) } \\
\hline Total & 188 & $15.22(13.78,16.85)$ & & $-1.29(-3.22,0.89)$ & $<0.001$ & \\
\hline Monotherapy & 26 & $16.00(14.36,16.25)$ & $0.565^{\mathrm{a}}$ & $-0.39(-0.90,1.35)$ & 0.753 & $0.050^{\mathrm{a}}$ \\
\hline Polytherapy & 162 & $15.00(13.72,16.94)$ & $0.946^{b}$ & $-2.13(-3.46,0.75)$ & $<0.001$ & $0.107^{\mathrm{b}}$ \\
\hline$A D+A A P$ & 30 & $15.23(14.33,17.53)$ & & $-2.20(-3.96,-1.01)$ & 0.006 & \\
\hline$A D+M S$ & 32 & $14.92(13.17,16.77)$ & & $-2.43(-3.09,-0.86)$ & 0.008 & \\
\hline $\mathrm{AAP}+\mathrm{MS}$ & 34 & $15.00(13.80,17.08)$ & & $-0.32(-3.68,2.66)$ & 0.408 & \\
\hline$A D+A A P+M S$ & 66 & $14.86(13.30,17.00)$ & & $-2.13(-3.48,1.03)$ & 0.007 & \\
\hline \multicolumn{7}{|l|}{ TT4 (nmol/L) } \\
\hline Total & 188 & $98.81(81.15,115.83)$ & & $-7.12(-22.80,11.03)$ & 0.007 & \\
\hline Monotherapy & 26 & $100.10(91.27,131.06)$ & $0.237^{\mathrm{a}}$ & $-1.00(-12.50,11.46)$ & 0.345 & $0.30 I^{\mathrm{a}}$ \\
\hline Polytherapy & 162 & $98.39(80.83,114.59)$ & $0.23 \mathrm{I}^{\mathrm{b}}$ & $-7.73(-25.11,11.06)$ & 0.007 & $0.644^{b}$ \\
\hline$A D+A A P$ & 30 & $92.12(76.73,109.72)$ & & $-7.19(-10.97,11.11)$ & 0.609 & \\
\hline$A D+M S$ & 32 & $106.30(94.33,122.45)$ & & $-I I .5 \mid(-25.01,10.75)$ & 0.109 & \\
\hline $\mathrm{AAP}+\mathrm{MS}$ & 34 & $100.12(78.30,113.24)$ & & $-9.47(-59.49,11.00)$ & 0.084 & \\
\hline$A D+A A P+M S$ & 66 & $90.51(81.98,105.37)$ & & $-7.04(-25.35,11.81)$ & 0.118 & \\
\hline \multicolumn{7}{|l|}{ CORT (nmol/L) } \\
\hline Total & 188 & $371.46(309.36,460.33)$ & & $-13.90(-86.66,85.65))$ & 0.031 & \\
\hline Monotherapy & 26 & $390.20(320.65,465.24)$ & $0.788^{\mathrm{a}}$ & $24.84(-133.44,143.00)$ & 0.650 & $0.857^{\mathrm{a}}$ \\
\hline Polytherapy & 162 & $370.80(298.02,458.15)$ & $0.992^{b}$ & $-14.70(-83.27,81.75)$ & 0.037 & $0.623^{b}$ \\
\hline$A D+A A P$ & 30 & $370.80(319.65,419.13)$ & & $-22.39(-85.5 I, 90.28)$ & 0.820 & \\
\hline$A D+M S$ & 32 & $390.65(288.98,470.85)$ & & $-60.80(-\mid 57.2 I, 35.4 I)$ & 0.030 & \\
\hline $\mathrm{AAP}+\mathrm{MS}$ & 34 & 391.71 (304.8I, 468.90) & & $5.84(-39.80,75.67)$ & 0.887 & \\
\hline$A D+A A P+M S$ & 66 & $368.70(293.90,472.50)$ & & $-13.10(-83.93,109.50)$ & 0.127 & \\
\hline
\end{tabular}

(Continued) 
Table 2 (Continued).

\begin{tabular}{|l|l|l|l|l|l|l|}
\hline & $\mathbf{N}$ & Baseline & $\mathbf{p}$ & Changes & $\mathbf{p}^{\mathbf{c}}$ & $\mathbf{p}$ \\
\hline ACTH (ng/L) & & & & & \\
Total & 188 & $34.60(22.38,4 I .50)$ & & $-2.00(-8.5 \mathrm{I}, 4.16)$ & 0.039 \\
Monotherapy & 26 & $32.83(18.38,40.33)$ & $0.55 \mathrm{I}^{\mathrm{a}}$ & $-2.74(-12.2 \mathrm{I}, 6.37)$ & 0.347 & $0.965^{\mathrm{a}}$ \\
Polytherapy & 162 & $34.91(22.43,4 I .70)$ & $0.490^{\mathrm{b}}$ & $-2.00(-8.58,4.24)$ & 0.178 & $0.118^{\mathrm{b}}$ \\
$\quad$ AD+AAP & 30 & $38.28(19.43,47.53)$ & & $-7.00(-18.18,-2.82)$ & 0.015 & \\
AD+MS & 32 & $29.60(19.98,38.1 \mathrm{I})$ & & $-2.24(-12.35,10.19)$ & 0.605 & \\
AAP+MS & 34 & $35.39(25.02,43.40)$ & & $-0.90(-3.67,8.43)$ & 0.836 \\
AD+AAP+MS & 66 & $34.12(20.95,40.34)$ & & $-2.00(-7.19,4.24)$ & 0.322 \\
\hline
\end{tabular}

Notes: ${ }^{a}$ Difference between the monotherapy group and the polytherapy group; 'Difference between the monotherapy group, the AD+AAP group, the AD+MS group, the $A A P+M S$ group, and the AD+AAP+MS group; 'Difference between baseline and after psychotropic drug treatment.

Abbreviations: TSH, thyroid-stimulating hormone, FT3, free triiodothyronine, TT3, total triiodothyronine; FT4, free thyroxine, TT4, total thyroxine, CORT, cortical hormone, ACTH, adrenocorticotrophic hormone; N, number; AD, antidepressant; AAP, atypical antipsychotic; MS, mood stabilizer.

with the severity of symptoms of $\mathrm{BD},{ }^{29}$ and the correlation between the decrease in cortisol hormone and the reduction of psychotic symptoms also confirms this view. ${ }^{30}$

Polypharmacy was more frequently used in patients with BD. In this study, polytherapy was used in $86.17 \%$ of participants. It was surprising that there were no significant difference in changes in serum TSH, FT3, TT3, FT4, TT4, ACTH, and CORT levels between psychotropic drug monotherapy and polytherapy. No correlations were found between those seven kinds of neuroendocrine hormones and the duration of treatment. In other words, changes in neuroendocrine hormones do not correlate with the type, amount, and duration of treatment of the psychotropic drugs used. However, a naturalistic study of severe mental disorders, including $\mathrm{BD}$, indicated that the use of antipsychotics in combination with other psychotropic medications, especially with antidepressants, resulted in lower FT4 levels compared to antipsychotics alone. ${ }^{31}$ Overall, there are relatively few studies on polypharmacy in BD, both in terms of the efficacy and the side effects of polypharmacy, and no consistent conclusions have been drawn for the time being. From the results of this study, there was no significant difference in the inhibitory effects of monotherapy and polytherapy on the HPA axis function and HPT axis function.

As far as we know, this is the first study to simultaneously compare changes in HPA axis function and HPT axis function in BD patients before and after psychotropic drug treatment. One major advantage of the present study compared to previous studies was that the assessment regarding HPA-HPT axes dysfunction was based on multiple indicators of abnormality rather than each measure individually, thus allowing comparison and correlation of findings between different axes.

There were some limitations of this study. First, the sample size of this study is relatively small, which is mainly due to the strict inclusion and exclusion criteria in this study. This affects the credibility of the findings to some extent, and therefore a larger sample size study is needed to validate the present results. Second, the psychotropic drugs referred to in this study only include the three classes-mood stabilizers, antidepressants, and atypical antipsychotics. In the statistical analysis, each class was analyzed as a whole, and no analysis was performed for a specific drug or a specific drug regimen. Thus, the effect of a specific drug or a specific medication regimen on neuroendocrine hormone levels in patients with $\mathrm{BD}$ is unclear. Finally, we did not count the doses of the corresponding drugs and could not assess the relationship between drug dose and neuroendocrine changes. However, whether there is a correlation between the dose of psychotropic drugs and the changes of neuroendocrine hormones is worth paying attention to, and further research is necessary.

\section{Conclusion}

Although the incidence of significant HPT axis and HPA axis dysfunction after psychotropic drug treatment is low in patients with BD who have normal baseline neuroendocrine hormone levels, psychotropic drug treatment does suppress HPT axis and HPA axis function. And this suppressive effect is not related to the use of psychotropic drugs alone or in combination, and is not related to the 
duration of psychotropic drug treatment. Therefore, even patients with BD who have normal baseline levels of neuroendocrine hormones should have their neuroendocrine hormone levels regularly monitored after psychotropic drug medication. But, the interval of monitoring can be slightly longer, which can be once every six months after taking psychotropic drugs.

\section{Abbreviations}

BD, bipolar disorder; ICD 10, International Classification of Diseases diagnostic Criteria, Edition 10; HPA, hypothalamic-pituitary-adrenal; HPT, Hypothalamic-thyroid; SSRIs, selective serotonin reuptake inhibitors; $\mathrm{TSH}$, thyroid-stimulating hormone; FT3, free triiodothyronine; TT3, total triiodothyronine; FT4, free thyroxine; TT4, total thyroxine; ACTH, adrenocorticotrophic hormone; CORT, cortical hormone; AD, antidepressant; AAP, atypical antipsychotic; MS, mood stabilizers.

\section{Ethics Approval}

The study was approved by the China Ethics Committee of Registering Clinical Trials. The code of ethics review is ChiECRCT-20180187. All procedures followed were in accordance with the ethical standards of the responsible committee on human experimentation and with the Helsinki Declaration of 1975, as revised in 2008 (5).

\section{Acknowledgments}

The authors are grateful to Dr. Jiajun Xu, Dr. Manxue Zhang, Dr. Jian Jiao, West China Hospital of Sichuan University, for the substantial work in the revision of the manuscript. Shengnan Zhao and Bo Zhang are co-first authors.

\section{Author Contributions}

All authors made substantial contributions to conception and design, acquisition of data, or analysis and interpretation of data; took part in drafting the article or revising it critically for important intellectual content; agreed to submit to the current journal; gave final approval of the version to be published; and agree to be accountable for all aspects of the work.

\section{Disclosure}

The authors have declared that no competing interests exist. The authors report no conflicts with any product mentioned or concept discussed in this article.

\section{References}

1. Alonso J, Petukhova M, Vilagut G, et al. Days out of role due to common physical and mental conditions: results from the WHO World Mental Health surveys. Mol Psychiatry. 2011;16:1234-1246. doi:10.1038/mp.2010.101

2. Martinez-Aran A, Vieta E, Torrent C, et al. Functional outcome in bipolar disorder: the role of clinical and cognitive factors. Bipolar Disord. 2007;9:103-113. doi:10.1111/j.1399-5618.2007.00327.x

3. Grande I, Goikolea JM, de Dios C, et al. Occupational disability in bipolar disorder: analysis of predictors of being on severe disablement benefit (PREBIS study data). Acta Psychiatr Scand. 2013;127:403-411. doi:10.1111/acps.12003

4. Pregelj P. Gene environment interactions in bipolar disorder. Psychiatr Danub. 2011;23(Suppl 1):S91-S93.

5. Serafini G, Canepa G, Adavastro G, et al. The relationship between childhood maltreatment and non-suicidal self-injury: a systematic review. Front Psychiatry. 2017;8:149. doi:10.3389/fpsyt.2017.00149

6. Serafini G, Gonda X, Canepa G, et al. Extreme sensory processing patterns show a complex association with depression, and impulsivity, alexithymia, and hopelessness. $J$ Affect Disord. 2017;210:249-257. doi:10.1016/j.jad.2016.12.019

7. Krishna VN, Thunga R, Unnikrishnan B, et al. Association between bipolar affective disorder and thyroid dysfunction. Asian J Psychiatr. 2013;6:42-45. doi:10.1016/j.ajp.2012.08.003

8. Ozsoy S, Mavili E, Aydin M, et al. Ultrasonically determined thyroid volume and thyroid functions in lithium-naïve and lithium-treated patients with bipolar disorder: a cross-sectional and longitudinal study. Hum Psychopharmacol. 2010;25:174-178. doi:10.1002/ hup. 1093

9. Fagiolini A. Medical monitoring in patients with bipolar disorder: a review of data. J Clin Psychiatry. 2008;69:e16. doi:10.4088/ jcp.0608e 16

10. Watson S, Gallagher P, Ritchie JC, et al. Hypothalamic-pituitaryadrenal axis function in patients with bipolar disorder. $\mathrm{Br}$ J Psychiatry. 2004;184:496-502. doi:10.1192/bjp.184.6.496

11. Daban C, Vieta E, Mackin P, et al. Hypothalamic-pituitary-adrenal axis and bipolar disorder. Psychiatr Clin North Am. 2005;28:469-480. doi:10.1016/j.psc.2005.01.005

12. Waldman SA, Park D. Myxedema coma associated with lithium therapy. Am J Med. 1989;87:355-356. doi:10.1016/s0002-9343(89) 80168-8

13. Zhang JX, Li X. Changes in serum thyroid hormone levels in psychiatric patients treated with second-generation antipsychotics. Endokrynol Pol. 2020;71:292-298. doi:10.5603/EP.a2020.0036

14. Kelly DL, Conley RR. Thyroid function in treatment-resistant schizophrenia patients treated with quetiapine, risperidone, or fluphenazine. J Clin Psychiatry. 2005;66:80-84. doi:10.4088/jcp.v66n0111

15. Gitlin M, Altshuler LL, Frye MA, et al. Peripheral thyroid hormones and response to selective serotonin reuptake inhibitors. J Psychiatry Neurosci. 2004;29:383-386.

16. de Carvalho GA, Bahls SC, Boeving A, et al. Effects of selective serotonin reuptake inhibitors on thyroid function in depressed patients with primary hypothyroidism or normal thyroid function. Thyroid. 2009;19:691-697. doi:10.1089/thy.2008.0261

17. Kopczak A, Stalla GK, Uhr M, et al. IGF-I in major depression and antidepressant treatment response. Eur Neuropsychopharmacol. 2015;25:864-872. doi:10.1016/j.euroneuro.2014.12.013

18. Knorr U, Vinberg M, Gether U, et al. The effect of escitalopram versus placebo on perceived stress and salivary cortisol in healthy first-degree relatives of patients with depression-A randomised trial. Psychiatry Res. 2012;200:354-360. doi:10.1016/j.psychres.2012.05.015

19. Handley R, Mondelli V, Zelaya F, et al. Effects of antipsychotics on cortisol, interleukin-6 and hippocampal perfusion in healthy volunteers. Schizophr Res. 2016;174:99-105. doi:10.1016/j. schres.2016.03.039 
20. Cohrs S, Röher C, Jordan W, et al. The atypical antipsychotics olanzapine and quetiapine, but not haloperidol, reduce ACTH and cortisol secretion in healthy subjects. Psychopharmacology. 2006;185:11-18. doi:10.1007/s00213-005-0279-x

21. Cole DP, Thase ME, Mallinger AG, et al. Slower treatment response in bipolar depression predicted by lower pretreatment thyroid function. Am J Psychiatry. 2002;159:116-121. doi:10.1176/appi. ajp.159.1.116

22. Chang KD, Keck PE, Stanton SP, et al. Differences in thyroid function between bipolar manic and mixed states. Biol Psychiatry. 1998;43:730-733. doi:10.1016/s0006-3223(98)80003-6

23. Ventura-Juncá R, Symon A, López P, et al. Relationship of cortisol levels and genetic polymorphisms to antidepressant response to placebo and fluoxetine in patients with major depressive disorder: a prospective study. BMC Psychiatry. 2014;14:220. doi:10.1186/ s12888-014-0220-0

24. Lin D, Mok H, Yatham LN. Polytherapy in bipolar disorder. CNS Drugs. 2006;20:29-42. doi:10.2165/00023210-200620010-00003

25. Reddy YJ, Jhanwar V, Nagpal R, et al. Prescribing practices of Indian psychiatrists in the treatment of bipolar disorder. Aust N Z J Psychiatry. 2019;53:458-469. doi:10.1177/0004867419826718
26. Gau CS, Chang CJ, Tsai FJ, et al. Association between mood stabilizers and hypothyroidism in patients with bipolar disorders: a nested, matched case-control study. Bipolar Disord. 2010;12:253-263. doi:10.1111/j.1399-5618.2010.00814.x

27. Li C, Lai J, Huang T, et al. Thyroid functions in patients with bipolar disorder and the impact of quetiapine monotherapy: a retrospective, naturalistic study. Neuropsychiatr Dis Treat. 2019;15:2285-2290. doi:10.2147/NDT.S196661

28. Subramaniam A, LoPilato A, Walker EF. Psychotropic medication effects on cortisol: implications for research and mechanisms of drug action. Schizophr Res. 2019;213:6-14. doi:10.1016/j.schres.2019.06.023

29. Belvederi Murri M, Pariante C, Mondelli V, et al. HPA axis and aging in depression: systematic review and meta-analysis. Psychoneuroendocrinology. 2014;41:46-62. doi:10.1016/j.psyneuen.2013.12.004

30. McKay MS, Zakzanis KK. The impact of treatment on HPA axis activity in unipolar major depression. $J$ Psychiatr Res. 2010;44:183-192. doi:10.1016/j.jpsychires.2009.07.012

31. Vedal TSJ, Steen NE, Birkeland KI, et al. Free thyroxine and thyroid-stimulating hormone in severe mental disorders: a naturalistic study with focus on antipsychotic medication. J Psychiatr Res. 2018;106:74-81. doi:10.1016/j.jpsychires.2018.09.014

\section{Publish your work in this journal}

Neuropsychiatric Disease and Treatment is an international, peerreviewed journal of clinical therapeutics and pharmacology focusing on concise rapid reporting of clinical or pre-clinical studies on a range of neuropsychiatric and neurological disorders. This journal is indexed on PubMed Central, the 'PsycINFO' database and CAS, and is the official journal of The International Neuropsychiatric Association (INA). The manuscript management system is completely online and includes a very quick and fair peer-review system, which is all easy to use. Visit http://www.dovepress.com/testimonials.php to read real quotes from published authors. 